\title{
Up-regulation of FTO gene expression was associated with increase in skeletal muscle mass in overweight male adolescents
}

\author{
Saeid Doaei ${ }^{1,2}$, Naser Kalantari ${ }^{3}$, Nastaran Keshavarz Mohammadi ${ }^{4}$, Pantea Izadi ${ }^{5}$, \\ Maryam Gholamalizadeh², Hassan Eini-Zinab³ ${ }^{3}$ Tuire Salonurmi ${ }^{6}$, Alireza Mosavi Jarrahi², \\ Shahram Rafieifar ${ }^{7}$, Reza Janipoor ${ }^{8}$, Mahnaz Sadeghypor ${ }^{9}$, Ghasem Azizi Tabesh ${ }^{5}$, Mark O. Goodarzi ${ }^{10}$
}

\author{
${ }^{1}$ Research Center of Health and Environment, Guilan University of Medical Sciences, \\ Rasht, Iran \\ ${ }^{2}$ Student Research Committee, Cancer Research Center, Shahid Beheshti University \\ of Medical Sciences, Tehran, Iran \\ ${ }^{3}$ Department of Community Nutrition, School of Nutrition and Food Sciences, Shahid \\ Beheshti University of Medical Sciences, Tehran, Iran \\ ${ }^{4}$ Department of Public Health, Shahid Beheshti University of Medical Sciences, \\ Tehran, Iran \\ ${ }^{5}$ Department of Medical Genetics, School of Medicine, Tehran University of Medical \\ Sciences, Tehran, Iran \\ ${ }^{6}$ Department of Internal Medicine, Oulu University Hospital and University of Oulu, \\ Oulu, Finland \\ ${ }^{7}$ Health Promotion and Education Department, Ministry of Health, Tehran, Iran \\ ${ }^{8}$ Department of Veterinary Medicine, School of Veterinary Medicine, Shiraz University \\ of Medical Sciences, Shiraz, Iran \\ ${ }^{9}$ Department of Food Science and Technology, Science and Research Branch, Islamic \\ Azad University, Tehran, Iran \\ ${ }^{10}$ Division of Endocrinology, Diabetes and Metabolism, Department of Medicine, \\ Cedars-Sinai Medical Center, Los Angeles, California, USA
}

Submitted: 25 February 2018

Accepted: 25 May 2018

Arch Med Sci 2019; 15 (5): 1133-1137

DOI: https://doi.org/10.5114/aoms.2019.87239

Copyright (c) 2019 Termedia \& Banach

\section{Abstract}

Introduction: The modifying effect of FTO gene expression level on change in body mass index and body composition has not been studied before. This study aimed to investigate the association between change in the expression level of the FTO gene and changes in anthropometric measurements in obese and overweight adolescent boys.

Material and methods: Eighty-four boys aged 12 to 16 years participated in this longitudinal study. A Bio Impedance Analyzer (BIA) was used to estimate percentage of body fat (\%BF) and percentage of skeletal muscle (\%SM). The FTO gene expression level in peripheral blood mononuclear cells was assessed using quantitative real-time PCR (qPCR). All measurements were performed at baseline and after 18 weeks.

Results: After 18 weeks, mean weight was reduced by $2.39 \mathrm{~kg}$, body mass index by $0.09 \mathrm{~kg} / \mathrm{m}^{2}$, \%BF by $0.82 \%$ and \%SM increased by $0.44 \%$. Moreover, the level of FTO gene expression increased 0.42 -fold higher than baseline. The change in expression level of the FTO gene was positively associated with change in \%SM ( $\beta=0.31, p=0.02)$.

Conclusions: FTO gene expression change was associated with change in $\% S M$ in male adolescents. Future studies are required to assess the interactions between FTO gene expression in different tissues and body composition.
Corresponding author: Maryam Gholamalizadeh Student Research Committee

Cancer Research Center

Shahid Beheshti

University of Medical Sciences

Tehran, Iran

Phone: 0216653265

E-mail: gholamalizadeh@ sbmu.ac.ir

Key words: FTO, gene expression, skeletal muscle, body fat, body mass index, obesity. 
S. Doaei, N. Kalantari, N. Keshavarz Mohammadi, P. Izadi, M. Gholamalizadeh, H. Eini-Zinab, T. Salonurmi, A. Mosavi Jarrahi, S. Rafieifar, R. Janipoor, M. Sadeghypor, G. Azizi Tabesh, M.O. Goodarzi

\section{Introduction}

Obesity in adolescents is the cause of many disorders is adulthood and has a genetic and environmental origin [1]. The FTO locus is one of the most important genetic risk factors for obesity in different populations [2]. There is no general agreement about the mechanism of the effects of the FTO locus on obesity. It is reported that the FTO polymorphisms play a key role in adjusting food and calorie intake; for example, people with AA and AT genotypes of the rs9939609 FTO polymorphism had $293 \mathrm{kcal} /$ day higher calorie intake compared to the people with TT genotype [3]. Moreover, people with FTO risk alleles have reduced lipolysis in adipocytes, suggesting a possible role of FTO in adipose tissue metabolism [4]. In other studies, a relationship between the levels of FTO gene expression in the hypothalamus and adipose tissue with body mass index (BMI) was reported $[5,6]$. Suppression of the FTO gene in rats resulted in reduction of the ratio of white adipose tissue to brown adipose tissue (WAT/BAT) [7]. However, the FTO gene is expressed ubiquitously [8], and the role of the FTO gene expression in other cells is not yet clear. For example, it is not clear whether the relationship between FTO gene expression and body weight and composition exists in all tissues or is exclusive to some tissues such as adipose and hypothalamus. Recent studies demonstrated a critical role of the FTO gene in cellular metabolic pathways associated with growth and proliferation. Overexpression of the FTO gene increases PI3K/AKT/mTOR pathway activity, which is important in regulating the cell cycle and consequently reduces apoptosis and allows proliferation [9]. Thus, the FTO gene might have a different role in cellular metabolism apart from its effect in adipocytes and the hypothalamus. For example, some recent human studies found an association of FTO gene expression level in peripheral blood mononuclear cells (PBMCs) with different metabolic pathways that affect BMI [10], ghrelin production [11], and the risk of type 2 diabetes [12].

Therefore, the aim of the present study was to investigate the relationship between changes in the FTO gene expression level in PBMCs with changes in body weight, $\mathrm{BMI}$ and body composition in adolescent boys after 18 weeks' intervention.

\section{Material and methods}

This study was an ancillary study within a randomized, controlled, school-based trial that carried out a comprehensive weight reduction program. This program consisted of personalized diet, physical activity and health education related to healthy nutrition and healthy weight for overweight and obese male adolescents. The details of the interventions were published previously [13]. The study involved students in two high schools $\left(7^{\text {th }}, 8^{\text {th }}\right.$, and $9^{\text {th }}$ grade students) of a district of Tehran city that was chosen randomly (district 5). In the original study, we had an intervention group $(n=44)$ and a control group $(n=40)$. However, in the present paper, we combined all subjects into a single group $(n=84)$. All study procedures were reviewed and approved by the ethics committee of Shahid Beheshti University of Medical Sciences, Tehran, Iran (reference number: Ir.sbmu.nnftri. rec.1394.22).

\section{Study population}

Ninety-six students expressed interest in participating in the ancillary study, and 84 enrolled and provided both baseline and week 18 blood samples.

The inclusion criteria were age between 12-16 years and $\mathrm{BMI} \geq+1$ z-scores. The specific exclusion criteria included: diagnosed weight-related diseases and use of weight-related medications.

The aims, methods and the other details of the project were explained to the participants and their parents, and written consent forms from both parents and students were obtained. All measures were taken between morning and noon at the baseline and after 18 weeks of the study.

\section{Anthropometric measures}

The measures of baseline and week 18 were obtained by trained personnel. The height of students was measured with a calibrated tape line fastened to a wall. A Bio Impedance Analyzer scale (Omron BF511, Kyoto, Japan) was used to measure weight, $\mathrm{BMI}$, percentage of body fat (\%BF), and percentage of skeletal muscle (\%SM) after entering their age, gender and height. This device is a digital, mobile and non-aggressive device that has eight electrodes that send an extremely weak electrical current of $50 \mathrm{kHz}$ and less than $500 \mu \mathrm{A}$ through the body to determine the amount of fat tissue. The validity of this device has been confirmed in a previous study [14]. All data were classified according to the z-score guidelines defined by WHO recommendations (for weight and $\mathrm{BMI}$ ) [15] and recently published papers (for \%BF and \%SM) [16].

\section{Gene expression}

At baseline and week 18, blood samples $(5 \mathrm{ml})$ were collected from all students who participated in the study, and then transferred to EDTA tubes and stored at $-80^{\circ} \mathrm{C}$. The PBMCs were isolated from anticoagulated peripheral blood by density centrifugation, and total RNA was subsequently isolated using the GeneAll RNA extraction kit (GeneAll, South Korea), cDNA synthesis was 
performed using cDNA synthesis kit (Gene All, South Korea), and mRNA expression levels were determined using the Opticon real-time PCR detection system (Bio-Rad Laboratories, California). Reactions were carried out in duplicate using SYBR Green Gene Expression Master Mix (Cat. no. 638317; Takara). The HPRT gene was chosen as a reference gene for its stable expression in PBMCs. Data were analyzed using the $2^{-\Delta \Delta C t}$ method and expressed as fold change [17].

\section{Statistical analysis}

Multiple linear regression was used to determine the relationship between baseline and changes in gene expression and baseline and change of anthropometric parameters after adjusting for age, physical activity, and calorie, protein and carbohydrate intake. Statistical analyses were performed using SPSS version 23.0 (IBM SPSS Statistics for Windows, IBM Corp., Armonk, NY, USA). The results were considered statistically significant at $p<0.05$.

\section{Results}

At baseline, subjects had a mean BMI of 26.4 $\pm 3.7 \mathrm{~kg} / \mathrm{m}^{2}$ (Table I). After 18 weeks, subjects experienced the following mean anthropometric parameter reductions: weight $2.4 \mathrm{~kg}, \mathrm{BMI} 0.09$ $\mathrm{kg} / \mathrm{m}^{2}$, and $\% \mathrm{BF} 0.82 \%$. Moreover, mean \%SM increased by $0.44 \%$, and the FTO expression was 0.42 -fold higher than baseline after 18 weeks.

At baseline, there was no significant association between FTO expression level and anthropometric measurements (Table II). The gene expression fold change was significantly associated with change in \%SM $(\beta=0.31, p=0.02)$. No significant association was detected between the change in FTO gene expression and change in other anthropometric indices (Table III).

\section{Discussion}

The results showed an association between up-regulation of FTO in PBMCs and increase in $\% S M$ at 18 weeks. The relationship between FTO gene expression and anthropometric measures has been reported in several studies. Berulava and Horsthemke reported that increased expression of FTO in PBMCs is associated with increased body mass [10].

Most of the studies on the interactions between FTO gene expression and body composition have focused on the expression level of the FTO gene in the hypothalamus and adipose tissue due to its hypothetical role in the regulation of energy intake and adipocyte differentiation. Church et al. reported that ubiquitous overexpression of FTO in the hypothalamus leads to a dose-dependent in- crease in body and fat mass [18]. The underlying mechanisms for the effect of FTO gene expression on body weight and body composition are not understood [18]. However, the role of FTO in the

Table I. Characteristics of the subjects $(N=84)$

\begin{tabular}{|lc|}
\hline Anthropometrics & Mean \pm SD \\
\hline Age [years] & $13.9 \pm 0.9$ \\
\hline Weight at baseline $[\mathrm{kg}]$ & $74.4 \pm 13.2$ \\
\hline Height at baseline $[\mathrm{cm}]$ & $167.1 \pm 8.5$ \\
\hline BMI at baseline $\left[\mathrm{kg} / \mathrm{m}^{2}\right]$ & $26.4 \pm 3.7$ \\
\hline BF at baseline $(\%)$ & $27.7 \pm 6.4$ \\
\hline SM at baseline $(\%)$ & $35.0 \pm 2.6$ \\
\hline Weight at 18 weeks $[\mathrm{kg}]$ & $72.0 \pm 11.9$ \\
\hline BMI at 18 weeks $\left[\mathrm{kg} / \mathrm{m}^{2}\right]$ & $26.3 \pm 3.67$ \\
\hline BF at 18 weeks $(\%)$ & $26.9 \pm 6.63$ \\
\hline SM at 18 weeks $(\%)$ & $35.48 \pm 2.66$ \\
\hline$\Delta$ Weight $[\mathrm{kg}]$ & $-2.4 \pm 4.1$ \\
\hline$\Delta$ BMI $\left[\mathrm{kg} / \mathrm{m}^{2}\right]$ & $-0.1 \pm 4.4$ \\
\hline$\Delta$ BF $(\%)$ & $-0.8 \pm 3.4$ \\
\hline$\Delta$ SM $(\%)$ & $0.4 \pm 2.8$ \\
\hline$\Delta$ FTO expression $\left(2^{-\Delta \Delta c t}\right)$ & \\
\hline
\end{tabular}

Table II. The association between FTO gene expression and anthropometric indices at baseline using linear regression adjusted for physical activity, and calorie, carbohydrate, protein and fat intake $(N=84)$

\begin{tabular}{|lcc|}
\hline Parameter & $\Delta$ FTO expression at baseline \\
\cline { 2 - 3 } & $\beta$ & $P$-value \\
\hline Weight & 0.05 & 0.74 \\
\hline BMI & 0.06 & 0.7 \\
\hline$\%$ BF & 0.15 & 0.37 \\
\hline \%SM & 0.17 & 0.31 \\
\hline
\end{tabular}

Table III. The association between FTO gene expression changes and changes in anthropometric indices using linear regression adjusted for physical activity, and calorie, carbohydrate, protein and fat intake $(N=84)$

\begin{tabular}{|lcc|}
\hline Parameter & \multicolumn{2}{c|}{ FTO expression fold changes } \\
\cline { 2 - 3 } & $\beta$ & $P$-value \\
\hline$\Delta$ Weight & 0.05 & 0.69 \\
\hline$\Delta \mathrm{BMI}$ & 0.01 & 0.92 \\
\hline$\Delta \% \mathrm{BF}$ & 0.13 & 0.64 \\
\hline$\Delta \% \mathrm{SM}$ & 0.31 & 0.02 \\
\hline
\end{tabular}


hypothalamus is likely different from its role in other tissues [19].

Recent studies have shown that FTO may have an essential role in metabolic-related signaling in most cells. For example, it is suggested that the FTO gene regulates the proliferation and differentiation of cells via PI3K/Akt signaling, and FTO knockdown leads to suppression of cell proliferation [9]. Moreover, more recent studies suggest that FTO acts as a mediator in the association between 5' AMP-activated protein kinase (AMPK) with the PI3K/AKT/mTOR pathway [20]. Such a critical role of the FTO gene may justify its ubiquitous expression.

However, it was observed that FTO overexpression reduces ghrelin mRNA and peptide levels and increases appetite [11]. Fischer et al. reported that the loss of function of the FTO gene in mice leads to a significant reduction in \%BF and lean body mass. They suggested that the leanness of FTO-deficient mice is due to increased energy expenditure and systemic sympathetic activation [19]. Moreover, FTO gene polymorphisms can modify the expression level of FTO and other BMI-related genes such as IRX3 [21].

In the present study, we did not find any significant relationship between FTO gene expression in PBMCs and weight, BMI and \%BF, which contrasts with the results of other studies on FTO gene expression and anthropometric parameters. FTO gene expression has been mainly assessed in obesity-related tissues such as the hypothalamus and adipose tissue. It has been strongly suggested that the role of FTO in the other tissues might be completely independent from its role in obesity [9]. The role of FTO gene expression in metabolic pathways related to cell growth and proliferation might be intensified in cells with a high metabolic rate (e.g., skeletal muscle cells). Moreover, it has been revealed that the relationship between FTO gene expression and \%BF and BMI can be influenced by FTO polymorphisms [10, 22]. Our separate study on the same subjects showed that the FTO gene polymorphism rs9930506 had a risk allele frequency of $40 \%$ and was strongly associated with \%BF and BMI [23]. Moreover, Hakanen et al. reported that the association between the FTO gene and BF and BMI could be altered at different ages [24, 25]. On the other hand, environmental factors such as lifestyle can play a key role in the interaction between FTO gene expression and anthropometric measures. For example, the level of FTO gene expression is influenced by dietary intake [26] and the effect of the FTO gene on obesity may be influenced by dietary components [27].

The present study had some limitations. This study was confined to male adolescents, and the results were reported regardless of the role of the FTO gene polymorphisms. It is possible that FTO gene ex- pression is influenced by gender, age and genotype. Future studies in both sexes, a broader age range and consideration of the FTO genotype are needed to address these limitations. We did not adjust the significance level for the number of tests performed because we did not want to limit the power of the study to discover novel associations. Thus, our results should be reproduced in independent cohorts.

In conclusion, this study did not find any significant association between the FTO gene expression and weight, BMI and \%BF. The up-regulation of the FTO gene expression in PBMCs that occurred during 18 weeks was positively associated with increase in \%SM in male adolescents. Future studies are required to assess the interactions between FTO gene expression and anthropometric measurements and whether these associations can be observed in all types of cells or is exclusive to specific cells.

\section{Acknowledgments}

We appreciate all the schools' staff for their excellent cooperation. The authors contributed equally to this work.

This study is related to the project NO. 1397/3904 from Student Research Committee, Shahid Beheshti University of Medical Sciences, Tehran, Iran. We also appreciate the "Student Research Committee" and "Research \& Technology Chancellor" in Shahid Beheshti University of Medical Sciences for their financial support of this study.

\section{Conflict of interest}

The authors declare no conflict of interest.

\section{References}

1. Ghomari-Boukhatem H, Bouchouicha A, Mekki K, Chenni K, Belhadj M, Bouchenak M. Blood pressure, dyslipidemia and inflammatory factors are related to body mass index in scholar adolescents. Arch Med Sci 2017; 13: 46-52.

2. Frayling TM, Timpson NJ, Weedon MN, et al. A common variant in the FTO gene is associated with body mass index and predisposes to childhood and adult obesity. Science 2007; 316: 889-94.

3. Speakman JR, Rance KA, Johnstone AM. Polymorphisms of the FTO gene are associated with variation in energy intake, but not energy expenditure. Obesity 2008; 16: 1961-5.

4. Almeida SM, Furtado JM, Mascarenhas P, et al. Anthropometric predictors of body fat in a large population of 9-year-old school-aged children. Obes Sci Prac 2016; 2: 272-81.

5. Klöting N, Schleinitz D, Ruschke K, et al. Inverse relationship between obesity and FTO gene expression in visceral adipose tissue in humans. Diabetologia 2008; 51: 641-7.

6. Fischer J, Koch L, Emmerling C, et al. Inactivation of the Fto gene protects from obesity. Nature 2009; 458: 894-8. 
7. Tews D, Fischer-Posovszky P, Wabitsch M. Regulation of FTO and FTM expression during human preadipocyte differentiation. Horm Metabol Res 2011; 43: 17-21.

8. Ulloa-Martínez M, Burguete-García Al, Murugesan S, Hoyo-Vadillo C, Cruz-Lopez M, García-Mena J. Expression of candidate genes associated with obesity in peripheral white blood cells of Mexican children. Arch Med Sci 2016; 12: 968-76.

9. Akbari ME, Gholamalizadeh M, Doaei S, Mirsafa F. FTO gene affects obesity and breast cancer through similar mechanisms: a new insight into the molecular therapeutic targets. Nutr Cancer 2018; 70: 30-6.

10. Berulava T, Horsthemke B. The obesity-associated SNPs in intron 1 of the FTO gene affect primary transcript levels. Eur J Hum Genet 2010; 18: 1054-6.

11. Karra E, O'Daly OG, Choudhury Al, et al. A link between FTO, ghrelin, and impaired brain food-cue responsivity. J Clin Investig 2013; 123: 3539-51.

12. Shen F, Huang W, Huang JT, et al. Decreased N 6-methyladenosine in peripheral blood RNA from diabetic patients is associated with FTO expression rather than ALKBH5. J Clin Endocrinol Metab 2015; 100: E148-54.

13. Kalantari N, Mohammadi NK, Rafieifar S, et al. Indicator for success of obesity reduction programs in adolescents: body composition or body mass index? Evaluating a school-based health promotion project after 12 weeks of intervention. Int J Prev Med 2017; 8: 73.

14. Bosy-Westphal A, Later W, Hitze B, et al. Accuracy of bioelectrical impedance consumer devices for measurement of body composition in comparison to whole body magnetic resonance imaging and dual $\mathrm{X}$-ray absorptiometry. Obesity Facts 2008; 1: 319-24.

15. Cole TJ, Flegal KM, Nicholls D, Jackson AA. Body mass index cut offs to define thinness in children and adolescents: international survey. BMJ 2007; 335: 194.

16. McCarthy HD, Samani-Radia D, Jebb SA, Prentice AM. Skeletal muscle mass reference curves for children and adolescents. Pediatr Obes 2014; 9: 249-59.

17. Pfaffl MW, Horgan GW, Dempfle L. Relative expression software tool $\left(\mathrm{REST}^{\circ}\right)$ for group-wise comparison and statistical analysis of relative expression results in real-time PCR. Nucleic Acids Res 2002; 30: e36.

18. Church C, Moir L, McMurray F, et al. Overexpression of FTO leads to increased food intake and results in obesity. Nat Genet 2010; 42: 1086-92.

19. Fischer J, Koch L, Emmerling C, et al. Inactivation of the FTO gene protects from obesity. Nature 2009; 458: 894-8.

20. Wu W, Feng J, Jiang D, et al. AMPK regulates lipid accumulation in skeletal muscle cells through FTO-dependent demethylation of N6-methyladenosine. Sci Rep 2017; 7: 41606.

21. Doaei S, Gholamalizadeh M, Jarrahi AM, Badakhanian $M$, Najafi R. The IRX3 gene: the missing link between the FTO gene and obesity. Asian Pac J Cancer Biol 2016; 1: 25-7.

22. Stratigopoulos G, Padilla SL, LeDuc CA, et al. Regulation of Fto/Ftm gene expression in mice and humans. Am J Physiol Regul Integr Comp Physiol 2008; 294: R1185-96.

23. Kalantari N, Mohammadi NK, Izadi P, et al. A haplotype of three SNPs in FTO had a strong association with body composition and BMI in Iranian male adolescents. PLOS One 2018; 13: e0195589.

24. Hakanen M, Raitakari OT, Ki TL, Peltonen N, Pahkala K. FTO genotype is associated with body mass index after the age of seven years but not with energy intake or leisure-time physical activity. J Clin Endocrinol Metab 2009; 94: 1281-7.
25. Jelavic MM, Babic Z, Pintaric $H$. The importance of two metabolic syndrome diagnostic criteria and body fat distribution in predicting clinical severity and prognosis of acute myocardial infarction. Arch Med Sci 2017; 13: 795-806.

26. Doaei S, Kalantari N, Mohammadi NK, Tabesh GA, Gholamalizadeh M. Macronutrients and the FTO gene expression in hypothalamus; a systematic review of experimental studies. Indian Heart J 2017; 69: 277-81.

27. Kalantari N, Doaei S, Keshavarz-Mohammadi N, Gholamalizadeh M, Pazan N. Review of studies on the fat mass and obesity-associated (FTO) gene interactions with environmental factors affecting on obesity and its impact on lifestyle interventions. ARYA Atheroscler 2016; 12: 281-90. 